DOI: 10.25712/ASTU.2072-8921.2018.01.018

УДК 536.46; 544.45; 614.83

\title{
ЧИСЛЕННОЕ ИССЛЕДОВАНИЕ ГОРЕНИЯ МЕТАНА В ЛАБОРАТОРНОЙ ТРУБЕ
}

\author{
М.О. Сысоева, Ю.А. Галенко, О.Б. Кудряшова, Е.В. Сыпин
}

Задача обеспечения безопасности производств, где возможно образование взрывоопасных газовых смесей, а также задача безопасного использования газового топлива в быту и на производстве стимулируют разработку средств и методов защиты объектов от взрывов газовых смесей. Для разработки методов предотвращения взрывов и средств подавления возгораний необходима информация о закономерностях возникновения и развития возгорания. Условия воспламенения и детонации взрывоопасных газовых смесей изучены достаточно полно, но работ, посвященных исследованию динамики процесса возгорания известно недостаточно. Между тем, знание времени индукции зажигания в зависимости от условий окружающей среды актуально для разработки систем мониторинга, защиты и предотвращения аварий.

Информация о динамике развития горения реакционноспособных газовых смесей может быть получена путём численного и натурного эксперимента.

Работа посвящена теоретическому исследованию процесса горения взрывоопасной газовой смеси в лабораторной трубе, влиянию кинетических параметров газовой смеси и параметров окружающей среды на данный процесс. Физико-математическая модель основана на уравнении теплопроводности и законе Аррениуса в одномерной постановке с потоком тепла заданной интенсивности в начале координат, и рассматривает стадии возникновения и распространения пламени. Разработана компьютерная модель, описывающая динамику процесса. В результате численного исследования модели получены зависимости температуры и скорости горения от времени. Предложена схема лабораторного стенда для экспериментального исследования горения метановоздушной смеси.

Ключевые слова: газовая смесь, горение, температура, тепловой поток, фрронт горения, моделирование, компьютерная модель, численное исследование, аналитическое решение, лабораторная труба, лабораторный стенд, датчик.

\section{ВВЕДЕНИЕ}

В связи с требованиями безопасности работ в шахтах, а также на производствах, где возможны выбросы взрывоопасных газовых смесей, не ослабевает интерес к моделированию горения и взрыва таких смесей. Химическая реакция в газах может распространяться в предельных режимах дозвукового распространения (со скоростями порядка нескольких сантиметров в секунду) и сверхзвуковой детонации (со скоростями на 3-4 порядка больше) [1-5]. Условия распространения таких волн определяются концентрационными и геометрическими пределами процесса, которые хорошо изучены [6].

Главный источник трагедий в шахтах это низкоскоростное горение метановых смесей, стационарный вариант которого хорошо изучен экспериментально. Менее изучены процессы перехода горения в детонацию, в силу большой сложности такого процесса. На практике для решения вопросов безопасности важно знать не только и не столько пределы детонации по концентрации и геомет- рии системы, сколько оценить скорость распространения пламени и время, проходящее от начала нагрева до детонации, в зависимости от условий протекания процесса и кинетических параметров смеси, а также саму возможность перехода волны горения в детонацию в данных условиях.

В обзоре [7] обобщены результаты экспериментальных исследований по влиянию условий процесса (температуры, давления, концентраций газовых реагентов) на скорость горения. Приближенная формула для расчета скорости горения предложена Зельдовичем и Франк-Каменецким еще в 1938 году [8]. Используя компьютерное моделирование, возможно не только рассчитать стационарную скорость горения газовой смеси, но рассмотреть процесс от нагревания газа до возможного перехода горения в детонацию.

В работе [9] с помощью компьютерного моделирования горения метановоздушных смесей на начальной стадии развития получены данные о зависимости радиуса сферического фронта горения от времени. Опреде- 
лены видимая скорость горения и нормальная скорость распространения пламени. Важной задачей остается решение вопроса о динамике процесса нестационарного горения метановоздушной смеси, в частности, изменения температуры смеси в процессе развития горения.

Цель данной работы - фризикоматематическое моделирование процесса нестационарного горения реакционноспособных смесей в одномерной постановке (лабораторной трубе) для прогнозирования зависимости температуры фрронта горения от времени.

\section{ТЕОРЕТИЧЕСКАЯ ЧАСТЬ}

Рассмотрим реакционноспособную газовую смесь, размещенную в полубесконечной трубе. В начале трубы действует тепловой поток плотности q. Будем считать коэффрициенты теплопроводности и диффузии равными; реакция в газовой смеси - первого порядка. Тогда в системе координат, связанной с фронтом волны горения, запишем:

$$
\begin{gathered}
c \rho\left(\frac{\partial T}{\partial t}+u \frac{\partial T}{\partial x}\right)=\frac{\partial}{\partial x}\left(\lambda \frac{\partial T}{\partial x}\right)+Q \rho w \\
w=\frac{\partial \eta}{\partial \tau}=(1-\eta) z e^{-\frac{E}{R T}}
\end{gathered}
$$

где $c, \rho, \lambda$ - удельная теплоемкость, плотность и теплопроводность газовой смеси, соответственно; П - глубина превращения; $u$ скорость горения; $Q$ - тепловой эффеект реакции на единицу массы смеси; $z$ - предэкспоненциальный множитель; $E$ - энергия активации; $w$ - скорость химической реакции.

Начальные и граничные условия для системы уравнений (1):

$$
\begin{aligned}
& t=0: T=T_{0}, \eta=0, u=0, \\
& x=0: q(t)=-\lambda \frac{d T}{d x}, \\
& x \rightarrow \infty: T=T_{0} .
\end{aligned}
$$

После завершения стадии воспламенения $t_{*}$, которую можно определить из условия $T(0, t *) \rightarrow T_{a} \quad$ (адиабатическая температура), фрронт горения начинает перемещаться со скоростью $и$ относительно начала трубы, и граничные условия запишутся в виде:

$$
\begin{aligned}
& x=0: T=T_{a}, \\
& x \rightarrow \infty: T=T_{0} .
\end{aligned}
$$

Для нахождения скорости горения можно воспользоваться уравнением Зельдовича [8]:

$$
u=\frac{1}{c \rho\left(T_{a}-T_{0}\right)} \sqrt{2 \lambda \int_{T_{a}}^{T_{0}} Q w(T) d T .}
$$

Другой способ поиска скорости горения подбор параметра $u$ в системе уравнений (1) с граничными условиями (3) такого, чтобы профили температуры и глубины превращения вещества, достигнутые на стадии воспламенения, оставались постоянными, то есть, при выходе на режим горения:

$$
t>t_{z}: T\left(x, t_{z}\right)=\text { const, } \eta\left(x, t_{z}\right)=\text { const. }
$$

Характерное время протекания химической реакции горения составит [10]:

$$
t_{a d}=\frac{\rho R T_{a}^{2}}{Q z E} \exp \left(E / R T_{a}\right) .
$$

Таким образом, система уравнений (1) с условиями (2) позволяет рассчитать профиль температуры в заданный момент времени, и динамику максимальной температуры на границе $x=0$.

\section{ЧИСЛЕННЫЕ ИССЛЕДОВАНИЯ}

Компьютерная модель исследуемого процесса использует аналитическое решение системы уравнений (1) с начальными и граничными условиями (2), (3).

При решении уравнения горения предполагалось, что $\eta=0$.

Поле температур $T(x, t)$ рассматривается в конечной области $0 \leq x \leq L, t>0$.

Дифференциальное уравнение в частных производных решается конечноразностным методом, при этом производные представляются разложением в ряды Тейлоpa.

Формируется двухмерная сетка узлов в поле температур. Для этого область $0 \leq x \leq L$ разделяется на $M$ равных частей с шагом $\Delta_{x}=\frac{L}{M} \quad$ и вводится обозначение $T(x, t)=T\left(i \Delta_{x}, j \Delta_{t}\right) \equiv T_{i}^{j}$.

Проводится дискретизация уравнения (1), используя центральные разности второго порядка точности для второй производной, первого порядка точности для первой производной по пространственной координате и разности вперед первого порядка для производной по времени. Получаем:

$$
\begin{aligned}
& c \rho \frac{T_{i}^{j+1}-T_{i}^{j}}{\Delta_{t}}+c \rho u_{i}^{j} \frac{T_{i+1}^{j}-T_{i-1}^{j}}{2 \Delta_{x}}= \\
& =\lambda \frac{T_{i-1}^{j}-2 T_{i}^{j}+T_{i+1}^{j}}{\Delta_{x}^{2}}+Q \rho w_{i}^{j}+O\left[\Delta_{t}, \Delta_{x}^{2}\right] .
\end{aligned}
$$

Уравнение (4) перегруппировывается и записывается в виде 


$$
\begin{gathered}
c \rho \frac{T_{i}^{j+1}}{\Delta_{t}}-c \rho \frac{T_{i}^{j}}{\Delta_{t}}+c \rho u_{i}^{j} \frac{T_{i+1}^{j}}{2 \Delta_{x}}-c \rho_{i}^{j} \frac{T_{i-1}^{j}}{2 \Delta_{x}}= \\
=\lambda \frac{T_{i-1}^{j}}{\Delta_{x}^{2}}-\lambda \frac{2 T_{i}^{j}}{\Delta_{x}^{2}}+\lambda \frac{T_{i+1}^{j}}{\Delta_{x}^{2}}+Q \rho w_{i}^{j}, \\
c \rho \frac{T_{i}^{j+1}}{\Delta_{t}}=\left(\frac{c \rho u_{i}^{j}}{2 \Delta_{x}}+\frac{\lambda}{\Delta_{x}^{2}}\right) T_{i-1}^{j}+\left(\frac{c \rho}{\Delta_{t}}-\frac{2 \lambda}{\Delta_{x}^{2}}\right) T_{i}^{j}+ \\
+\left(\frac{\lambda}{\Delta_{x}^{2}}-\frac{c \rho u_{i}^{j}}{2 \Delta_{x}}\right) T_{i+1}^{j}+Q \rho w_{i}^{j}, \\
T_{i}^{j+1}=\frac{\Delta_{t}}{c \rho}\left(\frac{c \rho u_{i}^{j}}{2 \Delta_{x}}+\frac{\lambda}{\Delta_{x}^{2}}\right) T_{i-1}^{j}+\frac{\Delta_{t}}{c \rho}\left(\frac{c \rho}{\Delta_{t}}-\frac{2 \lambda}{\Delta_{x}^{2}}\right) T_{i}^{j}+ \\
+\frac{\Delta_{t}}{c \rho}\left(\frac{\lambda}{\Delta_{x}^{2}}-\frac{c \rho u_{i}^{j}}{2 \Delta_{x}}\right) T_{i+1}^{j}+\frac{\Delta_{t}}{c \rho} Q \rho w_{i}^{j}, \\
T_{i}^{j+1}=\left(\frac{\Delta_{t}}{2 \Delta_{x}} u_{i}^{j}+\frac{\lambda \Delta_{t}}{c \rho \Delta_{x}^{2}}\right) T_{i-1}^{j}+\left(1-\frac{2 \lambda \Delta_{t}}{c \rho \Delta_{x}^{2}}\right) T_{i}^{j}+ \\
+\left(\frac{\lambda \Delta_{t}}{c \rho \Delta_{x}^{2}}-\frac{\Delta_{t}}{2 \Delta_{x}} u_{i}^{j}\right) T_{i+1}^{j}+\frac{Q \Delta_{t}}{c} w_{i}^{j}, \\
\text { где } \beta=\frac{\lambda \Delta_{t}}{c \rho \Delta_{x}^{2}}, \quad i=1,2, \ldots, M-1, \quad j=0,1, \ldots, \quad \text { с }
\end{gathered}
$$$$
\text { ошибкой усечения порядка } O\left[\Delta_{t}, \Delta_{x}^{2}\right] \text {. }
$$

Конечно-разностное приближение (8) диффференциального уравнения (1) включает только одно неизвестное значение температуры $T_{i}^{j+1}$ для временного уровня $j+1$, которое может быть непосредственно рассчитано из уравнения (8), когда известны узловые значения $T_{i-1}^{j}, T_{i}^{j}$ и $T_{i+1}^{j}$ на предыдущем временном слое $j$.

На рис. 1 схематично показано расположение узлов в рассматриваемой явной конечно-разностной схеме.

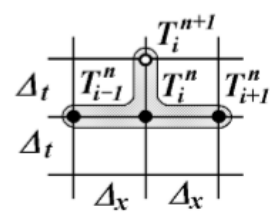

Рисунок 1 - Шаблон схемы с конечными разностями для простой явной схемы

При решении уравнения (8) формируется $\quad M-1$ алгебраическое уравнение, $i=1,2, \ldots, M-1$, содержащее $M+1$ неизвестных значений $T_{i}^{j+1}(i=0,1, \ldots, M)$. Из двух граничных условий для $i=0$ и $i=M$ получаются недостающие уравнения.

Граничные условия:

$$
\tau=0: T=T_{c p}, u=0 ;\left(T_{c p}\right. \text { - температура }
$$

окружающей среды)

$$
\begin{aligned}
& x=0:-\lambda \frac{\partial T}{\partial x}=q, u=0 ; \\
& x=M: \frac{\partial T}{\partial x}=0 .
\end{aligned}
$$

Дискретизируются граничные условия, используя формулу с центральными разностями второго порядка:

$$
x=0:-\lambda \frac{T_{i+1}^{j}-T_{i-1}^{j}}{2 \Delta_{x}}=q,
$$

при $i=0$ получаем $-\lambda \frac{T_{1}^{j}-T_{-1}^{j}}{2 \Delta_{x}}=q$,

$$
\begin{gathered}
T_{-1}^{j}=T_{1}^{j}+\frac{2 q}{\lambda} \Delta_{x} ; \\
x=M: \frac{T_{i+1}^{j}-T_{i-1}^{j}}{2 \Delta_{x}}=0,
\end{gathered}
$$

при $i=M$ получаем $\frac{T_{M+1}^{j}-T_{M-1}^{j}}{2 \Delta_{x}}=0$,

$$
T_{M+1}^{j}=T_{M-1}^{j} .
$$

Рассматриваются фриктивные узлы с индексом "-1" и фриктивной температурой $T_{-1}^{j}$ и индексом " $M+1$ " с фиктивной температурой $T_{M+1}^{j}$, которые получаются расширением вычислительной области на $\Delta_{x}$ налево $и$ направо, соответственно (рис. 2).

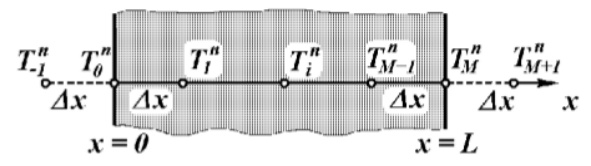

Рисунок 2 - Образование фиктивных узлов "-1" и " $M+1$ " с фриктивными температурами $T_{-1}^{j}$ и $T_{M+1}^{j}$

Два дополнительных соотношения, необходимые для устранения фриктивных температур, определяются после записывания уравнения (8) для $i=0$ и $i=M$ :

$$
\begin{aligned}
& T_{0}^{j+1}=\left(\frac{\Delta_{t}}{2 \Delta_{x}} u_{0}^{j}+\beta\right) T_{-1}^{j}+(1-2 \beta) T_{0}^{j}+ \\
& +\left(\beta-\frac{\Delta_{t}}{2 \Delta_{x}} u_{0}^{j}\right) T_{1}^{j}+\frac{Q \Delta_{t}}{c} w_{0}^{j}, \\
& T_{M}^{j+1}=\left(\frac{\Delta_{t}}{2 \Delta_{x}} u_{M}^{j}+\beta\right) T_{M-1}^{j}+(1-2 \beta) T_{M}^{j}+ \\
& +\left(\beta-\frac{\Delta_{t}}{2 \Delta_{x}} u_{M}^{j}\right) T_{M+1}^{j}+\frac{Q \Delta_{t}}{c} w_{M}^{j} .
\end{aligned}
$$


Затем $T_{-1}^{j}$ устраняется с помощью уравнений (9) и (11), $T_{M+1}^{j}$ устраняется с помощью уравнений (10) и (12). В результате получаем:

$$
\begin{aligned}
& T_{0}^{j+1}=(1-2 \beta) T_{0}^{j}+2 \beta T_{1}^{j}+ \\
+ & \frac{q}{\lambda}\left(\Delta_{t} u_{0}^{j}+2 \beta \Delta_{x}\right)+\frac{Q \Delta_{t}}{c} w_{0}^{j}, \\
T_{M}^{j+1}= & 2 \beta T_{M-1}^{j}+(1-2 \beta) T_{M}^{j}+\frac{Q \Delta_{t}}{c} w_{M}^{j} .
\end{aligned}
$$

Уравнения с конечными разностями (8) вместе с уравнениями (13) и (14) формируют $M+1$ уравнений для определения неизвестных температур в узлах на каждом последующем временном слое и являются численной моделью процесса горения в одномерной пластине.

Чтобы организовать решение одномерной нестационарной задачи горения конечноразностным методом по явной схеме, выбирается шаг временной дискретизации, величина которого определяет значение параметра $\beta=\frac{\lambda \Delta_{t}}{c \rho \Delta_{x}^{2}}$, где $\Delta_{t}$ - шаг временной дискретизации, $\Delta_{x}^{2}-$ шаг пространственной дискретизации задачи.

Для анализа устойчивости в некоторый узел с индексами $(i, j)$ вносится малое возмущение $\varepsilon_{i}^{j}$. Тогда применение вычислительной схемы (8) приводит к тому, что после проведения итераций в узле с индексами $(i, j+1)$ появляется значение, отличающееся от решения, которое появилось бы в случае отсутствия возмущения $\varepsilon_{i}^{j}$.

Величина возмущения, внесенного в узел $(i, j)$, оценивается из выражения

$$
\begin{aligned}
& \left(T_{i}^{j+1}+\varepsilon_{i}^{j+1}\right)=\left(\frac{\Delta_{t}}{2 \Delta_{x}} u_{i}^{j}+\beta\right) T_{i-1}^{j}+ \\
& +(1-2 \beta)\left(T_{i}^{j}+\varepsilon_{i}^{j}\right)+\left(\beta-\frac{\Delta_{t}}{2 \Delta_{x}} u_{i}^{j}\right) T_{i+1}^{j}+\frac{Q \Delta_{t}}{C_{P}} w_{i}^{j}
\end{aligned}
$$

Это уравнение вычитается из уравнения (8) и определяется величина возмущения, появившаяся на следующем временном слое

$$
\varepsilon_{i}^{j+1}=(1-2 \beta) \varepsilon_{i}^{j} .
$$

Математически условие устойчивости представляется в виде

$$
\left|\frac{\varepsilon_{i}^{j+1}}{\varepsilon_{i}^{j}}\right|<1, \text { или }|1-2 \beta|<1 .
$$

Решая неравенство, получаются условия статической $\Delta_{t}>0$ и динамической устойчивостей $\Delta_{t}<\frac{C_{P} \rho}{\lambda} \Delta_{x}^{2}$.

Если учесть, что начальное возмущение, внесенное в узел $(i, j)$, передается не только в узел $(i, j+1)$, но и в узлы $(i+1, j+1)$ и $(i-1, j+1)$, то после преобразований получается:

$$
0<\beta<0,5 \text {, т.e. } \Delta_{t}^{\max }=\frac{C_{P} \rho}{2 \lambda} \Delta_{x}^{2},
$$

где $\Delta_{t}^{\max }$ - максимальная величина шага по времени $\Delta_{t}$, в пределах которого схема является устойчивой.

\section{РЕЗУЛЬТАТЫ И ОБСУЖДЕНИЕ}

Для расчета реакции метана с воздухом были выбраны параметры, приведенные в [7]: $Q=50,125 \quad$ МДж/кг, $E=0,238 \quad$ МДж/моль, $z=9,66 \cdot 10^{10} \mathrm{c}^{-1}$. Теплофизические параметры смеси рассчитывались по стехиометрическому соотношению и равнялись: $c=1133$ Дж/(кг'К), $\quad \lambda=0,026 \quad$ Дж/(м'К'с), $\rho=1,22$ кг/M ${ }^{3}$. Адиабатическая температура $T_{a}=1950^{\circ} \mathrm{C}$. Характерное время химической реакции, рассчитанное по формуле (6), составит $t_{a d}=6,4 \cdot 10^{-10} \mathrm{c}$.

Время индукции зажигания, в зависимости от плотности теплового потока, исчисляется секундами и десятками секунд. Задача имеет пограничный характер по времени: сначала происходит разогрев смеси, практически, по линейному закону, затем - в течение крайне короткого времени - резкий скачок температуры до максимальной (рис. 3).

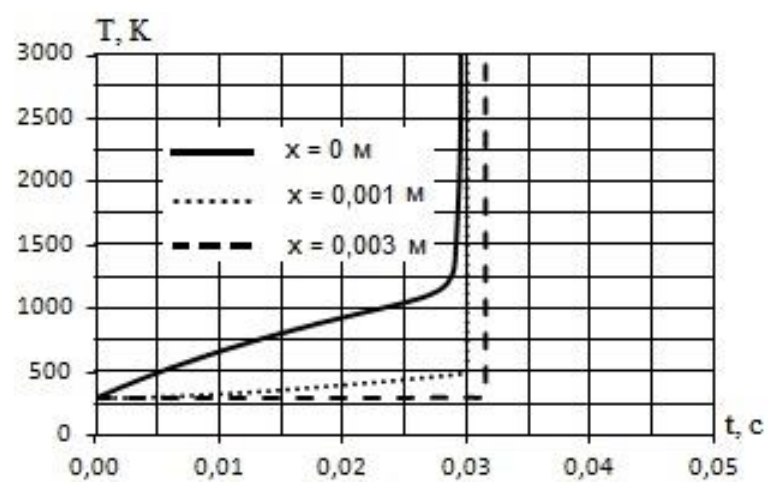

Рисунок 3 - Изменение температуры при инициировании метановоздушной смеси тепловым потоком плотностью $30 \cdot 10^{3} \mathrm{BT} / \mathrm{M}^{2}$ для разных сечений лабораторной трубы 
Таким образом, задача имеет пограничный характер по времени: сначала происходит разогрев смеси, практически, по линейному закону, затем - в течение крайне короткого времени - резкий скачок температуры до максимальной.

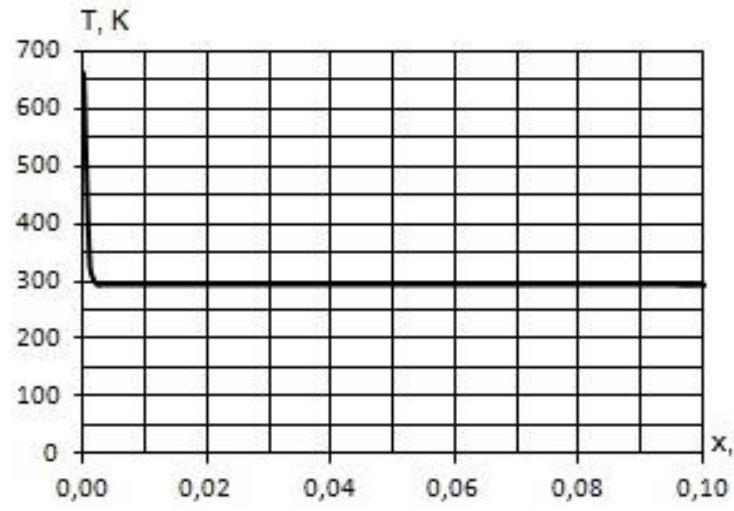

Рисунок 4 - Изменение температуры при инициировании метановоздушной смеси тепловым потоком плотностью $30 \cdot 10^{3}$ Вт/м² в момент времени $t=0,01 \mathrm{C}$

Скорость нормального горения, рассчитанная по формуле (4), составляет, примерно, 0,52-0,53 м/с и растет линейно в зависимости от начальной температуры.

Исследование зависимости температуры горения метановоздушной смеси от координаты трубы показывает, что максимальное значение температуры наблюдается в начале трубы, затем зависимость характеризуется резким падением температуры до температуры окружающей среды (рис.4).

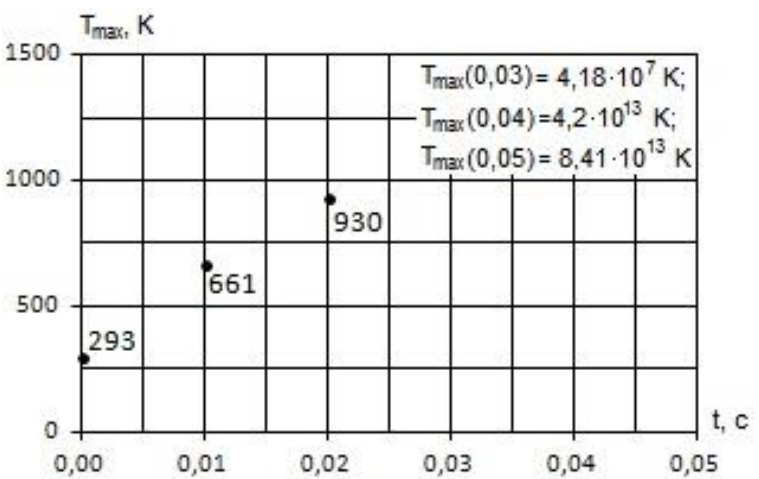

Рисунок 5 - Изменение максимальной температуры горения метановоздушной смеси от времени

На рисунке 5 представлена зависимость максимальной температуры горения метановоздушной смеси, рассчитанная в трубе в данный момент времени, в зависимости от времени. Сначала максимальная температу- ра растет практически линейно, затем наблюдается резкий скачок температуры (свыше 1000 K).

Время индукции зажигания $t_{z}$ существенно зависит от плотности теплового потока на границе (рис. 6 , в качестве $t$ z оценивалось время, при котором температура достигала $\left.T_{a}\right)$.

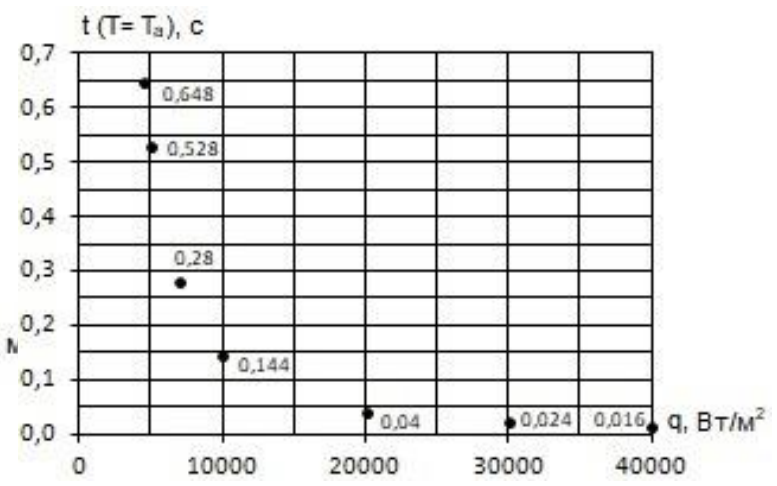

Рисунок 6 - Время индукции зажигания метановоздушной смеси в зависимости от плотности теплового потока на границе

Время индукции зажигания $t_{z}$ слабо зависит от начальной температуры. Учет выгорания дает поправку не более 3-4 \% в расчет времени индукции.

\section{ЭКСПЕРИМЕНТАЛЬНАЯ ЧАСТЬ}

Для проведения экспериментальных исследований процесса горения метановоздушной смеси предложена следующая схема лабораторного стенда:

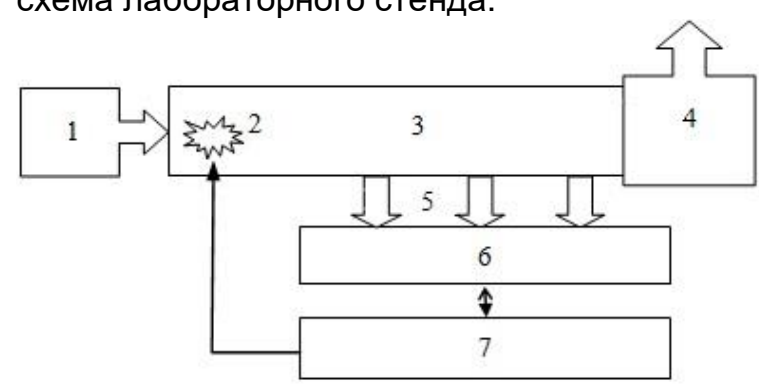

1 - баллон с газом, 2 - система

воспламенения, 3- труба с газо-воздушной смесью, 4 - вытяжная вентиляция,

5 - датчики, 6 - регистратор, 7 - система синхронизации

Рисунок 7 - Схема стенда

Следующим этапом работы предполагается проведение исследований времени индукции зажигания, температуры и скорости горения метановоздушной смеси в 
зависимости от плотности теплового потока на границе и температуры окружающей среды.

\section{ВЫВоды}

Таким образом, в работе проведено математическое моделирование процесса нестационарного горения реакционноспособной газовой смеси в одномерной постановке (лабораторной трубе). На примере метановоздушной смеси определены значимые факто ры, влияющие на время индукции зажигания: плотность теплового потока, начальная температура. Установлено, что учет выгорания смеси слабо влияет на результат расчетов. Определена скорость нормального горения и характерное время протекания химической реакции.

Предложено численное решение задачи определения скорости горения метановоздушной смеси применительно к лабораторной установке.

Исследовано влияние кинетических параметров газовой смеси и параметров окружающей среды на скорость фронта горения.

Предложена схема лабораторного стенда для экспериментального исследования процесса.

\section{СПИСОК ЛИТЕРАТУРЫ}

1. Зельдович Я.Б., Компанеец А.С. Теория детонации. - Москва: Гостехиздат, 1955.

2. Щелкин К.И., Трошин Я.К. Газодинамика горения. - М.: из-во АН СССР, 1963. - 256 с.

3. Войцеховский Б.В., Митрофранов В.В., Топчиян М.Е. Структура фронта детонации в газах. Новосибирск: из-во СО АН СССР, 1963. - 168 с.

4. Физика взрыва /под ред. Л.П.Орленко. - М.: Физматлит, 2002. - 832 с.

5. Льюис Б., Эльбе Г. Горение, пламя и взрывы в газах. - М.: Мир, 1984. - 448 с.

6. Бунев В.А., Коржавин А.А., Сеначин П.К. Анализ влияния различных фракторов на характеристики взрывоопасности метана // Ползуновский вестник. - 2012. - № 3/1. - с. 5-16.

7. Законы горения / Под ред. Ю.В. Полежаева.- М.: УНПЦ «Энергомаш», 2006. - 351 с.

8. Зельдович Я.Б., Франк-Каменецкий Д.А. Теория теплового распространения пламени // Ж. Физ. Химии. - 1938, № 12. - с. 100.-105.
9. Лисаков С.А., Сидоренко А.И., Павлов А.Н., Сыпин Е. В., Леонов Г. В. Компьютерное моделирование горения метано-воздушных смесей на начальной стадии развития // Вестник научного центра по безопасности работ в угольной промышленности. - 2016. - № 3. - с. 32-41.

10. Зельдович Я.Б., Баренблатт Г.Ш., Либрович В.Б. и др. Математическая теория горения и взрыва. - М.: Наука, 1980. - 478 с.

Сысоева Маргарита Олеговна, кандидат физико-математических наук, кафедра естественнонаучных дисциплин Бийского технологического института (филиала) федерального государственного бюджетного образовательного учреждения высшего образования «Алтайский государственный технический университет им.И.И. Ползунова», e-mail: sysoeva.mo@bti.secna.ru; 8-923-652-1248.

Галенко Юрий Анатольевич, доктор фризико-математических наук, профессор, кафредра естественнонаучных дисциплин Бийского технологического института (филиала) федерального государственного бюджетного образовательного учреждения высшего образования «Алтайский государственный технический университет им. И.И. Ползунова», e-mail: gal@bti.secna.ru; 8-902-141-5775.

Кудряшова Ольга Борисовна, доктор фризико-математических наук, профрессор, кафредра ракетных двигателей и высокоэнергетических устройств автоматических систем Бийского технологического института (филиала) федерального государственного бюджетного образовательного учреждения высшего образования «Алтайский государственный технический университет им.И.И. Ползунова», e-mail: obk@bti.secna.ru;

Сыпин Евгений Викторович, кандидаm технических наук, профессор, кафедра методов и средств измерений и автоматизации Бийского технологического института (филиала) федерального государственного бюджетного образовательного учреждения высшего образования "Алтайский государственный технический универcumem им.И.И. Ползунова», e-mail: sev@bti.secna.ru. 